\title{
Desempenho de métodos de estimativa da evapotranspiração de referência nas localidades de Frederico Westphalen e Palmeira das Missões, RS
}

\author{
Perfomance of methods for estimating reference evapotranspiration in the municipalities of Frederico \\ Westphalen and Palmeira das Missões, State of Rio Grande do Sul, Brazil
}

\author{
Felipe Gustavo Pilau ${ }^{{ }^{*}}$ Rafael Battisti $^{\text {II }}$ Lucindo Somavilla ${ }^{\text {III }}$ Evandro Zanini Righi ${ }^{\mathrm{I}}$
}

\section{RESUMO}

A estimativa da evapotranspiração de referência (ETo), usada no balanço hídrico, possibilita quantificar o armazenamento de água no solo, auxiliando no manejo da irrigação. O objetivo do trabalho foi comparar métodos mais simples de estimativa da evapotranspiração de referência com o método Penman-Monteith (FAO), nas escalas diária e de 5, 10, 15 e 30 dias, e mensal, para os municípios de Frederico Westphalen e Palmeira das Missões, no RS. Os métodos avaliados tenderam a melhorar a eficiência com o aumento da escala temporal de estudo, mantendo o mesmo desempenho para ambas as localidades. Os maiores e menores valores de ETo ocorreram nos meses de dezembro e junho, respectivamente. A maioria dos métodos subestimou os valores de ETo. Em qualquer escala temporal, os métodos de Makking e da Radiação FAO24 podem substituir o modelo de PenmanMonteith.

Palavras-chave: elementos climáticos, métodos empíricos, evapotranspiração.

\section{ABSTRACT}

The estimation of reference evapotranspiration (ETo), used in water balance, allows to determine soil water content, assisting on irrigation management. The present study aimed to compare simple ETo estimating methods with the Penman-Monteith (FAO), in the folowing time scales: daily, 5, 10, 15 and 30 days and monthly in the counties of Frederico Westphalen and Palmeira das Missões, in the Rio Grande do Sul state, Brazil. The methods tested had their efficiency improved by increasing the time scale of analysis, keeping the same performance for both locations. The highest and lowest ETo values occurred in December and June, respectively. Most methods underestimated ETo. For any of the time scales Makking and Radiaton FAO24 methods can replace the Penman-Monteith for estimating ETo.

Key words: climatic elements, empirical methods, evapotranspiration.

\section{INTRODUÇÃO}

As condições meteorológicas no Rio Grande do Sul são influenciadas pelos fenômenos El Niño e La Niña, alterando o regime das chuvas do Estado e interferindo na produção agrícola (BERLATO et al., 2005). Na safra 2004/05, devido à estiagem provocada pelo fenômeno La Niña, a produtividade média foi de apenas $630 \mathrm{~kg} \mathrm{ha}^{-1}$ de soja e $1.270 \mathrm{~kg} \mathrm{ha}^{-1}$ de milho (CONAB, 2009), acentuando as discussões sobre viabilidade de uso de irrigação nessas culturas.

O manejo da irrigação em culturas agrícolas passa fundamentalmente pela determinação da sua evapotranspiração. Como alternativa mais simples para determinar a evapotranspiração da cultura (ETc), podese adotar o procedimento sugerido pela FAO (ALLEN et al., 1998), multiplicando-se a evapotranspiração de referência (ETo) e o coeficiente de cultura (Kc).

Dentre os métodos de estimativa da ETo, o de Penman-Monteith (FAO) (ALLEN et al., 1998) exige

'Departamento de Fitotecnia, Centro de Ciências Rurais (CCR), Universidade Federal de Santa Maria (UFSM), 97105-900, Santa Maria, RS, Brasil. E-mail: fgpilau@smail.ufsm.br.*Autor para correspondência.

IPrograma de Pós-graduação em Engenharia de Sistemas Agrícolas, Escola Superior de Agricultura "Luiz de Queiroz (ESALQ), Universidade de São Paulo (USP), Piracicaba, SP, Brasil.

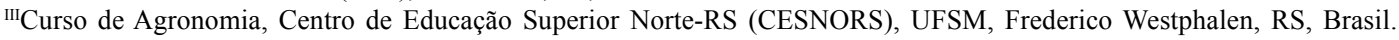


parâmetros de entrada nem sempre medidos em estações agrometeorológicas, tornando-o de uso restrito para a maioria das condições. Assim, é recomendável que estudos que avaliem o desempenho de métodos mais simples sejam feitos.

Dos métodos que exigem apenas a medida da temperatura do ar, CAMARGO \& SENTELHAS (1997), ao avaliarem os resultados com base no coeficiente de confiança ou desempenho "c", obtiveram classificação muito boa para os métodos de Camargo, Thornthwaite e Thornthwaite modificado por Camargo, enquanto SILVA et al. (2005) ressaltam subestimativas da ETo pelos métodos de Thornthwaite e Camargo em relação a Penman-Monteith, em clima subtropical úmido.

Para Bento Gonçalves-RS, Thornthwaite $(\mathrm{c}=0,58)$ e Camargo $(\mathrm{c}=0,61)$ apresentaram desempenho regular, enquanto Thornthwaite modificado por Camargo $(c=0,78)$ obteve desempenho muito bom em comparação a Penman-Monteith (CONCEIÇÃO \& MANDELLI, 2005). Para Santa Maria-RS, o método de Camargo apresentou desempenho muito bom $(c=0,79)$ (MEDEIROS, 1998).

Outro método que também utiliza apenas a medida da temperatura do ar é o de HARGREAVES \& SAMANI (1985), com bons resultados para Santa Maria-RS (c=0,73) (MEDEIROS, 1998) e Bento Gonçalves-RS $(\mathrm{c}=0,84)$ (CONCEIÇÃO \& MANDELLI, 2005). Para Palotina, no PR, com $\mathrm{c}=0,76$, o método de Hargreaves \& Samani, juntamente aos de Thornthwaite e Camargo, apresentaram bom desempenho em comparação a Penman-Monteith (FAO) (SYPERRECK et al., 2008). Para a região do Baixo Rio Grande, no noroeste do Estado de São Paulo, o método de Hargreaves \& Samani $(\mathrm{c}=0,82)$, juntamente ao método de Thornthwaite modificado, apresentaram os melhores desempenhos em comparação a Penman-Monteith (FAO) (CONCEIÇÃO, 2003).

Dispondo-se de medida da temperatura do ar e radiação solar ou insolação, pode-se utilizar o método de Makking. Segundo CONCEIÇÃO \& MANDELLI (2005), em Bento Gonçalves-RS, para substituir o padrão Penman-Monteith (FAO), esse é um dos métodos preferenciais. Ainda para o RS, MEDEIROS (2003) obteve um bom resultado do método de Makking $(\mathrm{c}=0,72)$, porém aquém ao método de Camargo, que utiliza apenas a medida da temperatura do ar. Nas regiões Norte, Sul e Serrana do Espírito Santo, conforme REIS et al. (2007), para períodos de 3, 5 e 7 dias, o método de Makking apresentou desempenho bom a muito bom, ainda que aquém aos métodos de Penman (48) Original, Penman Modificado (FAO 24), Pristley-Taylor, Blaney-Criddle (FAO 24) e Turc (1961).
Tendo ainda dados médios de umidade relativa do ar e velocidade do vento, além de temperatura do ar e radiação solar, é possível a adoção do método da Radiação-FAO, sugerido por CONCEIÇÃO \& MANDELLI (2005) para estimativa da ETo para Bento Gonçalves-RS. REIS et al. (2007), para as regiões Norte, Sul e Serrana do Estado do Espírito Santo, destacam que, dentre outros, o método da Radiação-FAO apresentou os melhores resultados, ainda que preferencialmente devam ser utilizados os métodos Penman (48) Original, Penman Modificado (FAO 24), Pristley-Taylor, Blaney-Criddle (FAO 24) e Turc (1961).

Dispondo de inúmeros métodos para estimativa da ETo, e sabendo dos diferentes níveis de desempenho de cada um frente a distintas condições climáticas, o trabalho objetivou comparar alguns métodos de estimativa de evapotranspiração de referência, com o método de Penman-Monteith, para as localidades de Frederico Westphalen e Palmeira das Missões, situados na região norte do Rio Grande do Sul, em diferentes escalas temporais.

\section{MATERIAL E MÉTODOS}

Os dados de temperatura e umidade relativa do ar, velocidade do vento a $10 \mathrm{~m}$ de altura e radiação solar global (Rs) foram obtidos, na escala horária, junto às estações do Instituto Nacional de Meteorologia, localizadas nos campi do Centro de Educação Superior Norte-RS, da Universidade Federal de Santa Maria, nos municípios de Frederico Westphalen $\left(27^{\circ} 23^{\prime} \mathrm{S}, 5^{\circ} 25^{\prime} \mathrm{O}\right.$ e 490m) e Palmeira das Missões (27 $55^{\circ} \mathrm{S}, 5^{\circ} 19^{\prime} \mathrm{O}$ e $620 \mathrm{~m})$. O clima dessa região é classificado como subtropical úmido, tipo Cfa2 (MORENO, 1961).

Para a determinação do saldo de radiação total diário $(\mathrm{Rn})$, foi utilizada a equação $\mathrm{Rn}=0,6011$. $\mathrm{Rs}$ $\left(\mathrm{R}^{2}=0,9226\right)$, gerada a partir de um ano de medidas realizadas nas estações meteorológicas e correlações entre as variáveis. A velocidade do vento a $2 \mathrm{~m}$ de altura foi obtida a partir da equação U2=Uz.(2/z). ${ }^{0,2}$ (PEREIRA et al., 1997).

As séries de dados meteorológicos totalizam dois anos de coleta ininterrupta (01/01/2008 a $31 / 12 / 2009$ ). Foram usados os dados integrados na escala diária e nas escalas de 5, 10, 15 e 30 dias para avaliação do desempenho dos métodos de estimativa da evapotranspiração de referência (ETo). Dias chuvosos foram descartados, realizando-se um agrupamento dos dados restantes, não mais seguindo o calendário Juliano, restando menos de 365 dias de dados por ano. Para a análise do desempenho dos métodos nas diferentes estações do ano, um novo agrupamento dos dados foi realizado, resultando num 
número variado de dias úteis por mês, somando-se os dois anos de avaliações (Tabela 1). Para essa segunda análise, foram utilizados os dados médios mensais.

Para a comparação entre os métodos de estimativa da ETo, expressa em $\mathrm{mm} \mathrm{dia}^{-1}$, o método de Penman-Monteith (FAO) (EToPM) foi tomado como referência (ALLEN et al., 1998), conforme Eq. (1):

$$
\mathrm{EToPM}=\frac{0,408 \cdot s \cdot(\mathrm{Rn}-\mathrm{G})+\frac{\gamma \cdot 900 \cdot \mathrm{U}_{2} \cdot(\mathrm{es}-\mathrm{ea})}{\mathrm{T}+273}}{\mathrm{~s}+\gamma \cdot\left(1+0,34 \cdot \mathrm{U}_{2}\right)}
$$

em que: $\mathrm{s}$ é a declividade da curva de pressão de vapor em relação à temperatura $\left(\mathrm{kPa}{ }^{\circ} \mathrm{C}\right) ; \mathrm{Rn}$ é o saldo de radiação diário $\left(\mathrm{MJ} \mathrm{m}^{-2} \mathrm{~d}^{-1}\right)$; $\mathrm{G}$ é o fluxo total diário de calor no solo, assumindo-se valor igual a zero; $\gamma$ é o coeficiente psicrométrico (valor constante de 6,215.10-2 $\left.\mathrm{kPa}{ }^{\circ} \mathrm{C}\right) ; \mathrm{U}_{2}$ é a velocidade do vento a $2 \mathrm{~m}$ de altura $\left(\mathrm{m} \mathrm{s}^{-1}\right)$; $\mathrm{e}_{\mathrm{s}}$ é a pressão de saturação de vapor $(\mathrm{kPa})$; $\mathrm{e}_{\mathrm{a}}$ é a pressão atual de vapor $(\mathrm{kPa})$; e T a temperatura média do $\operatorname{ar}\left({ }^{\circ} \mathrm{C}\right)$.

Como métodos simples alternativos, foram empregados os seguintes:

a) Método de Thornthwaite (EToTh) (THORNTHWAITE, 1948), conforme Eq. (2):

$\mathrm{EToTh}=16 .(10 . \mathrm{T} / \mathrm{I})^{\mathrm{a}} .(\mathrm{N} / 12 . \mathrm{ND} / 30)$

em que: T é a temperatura média diária $\left({ }^{\circ} \mathrm{C}\right)$; I é o índice de calor da região; a uma função cúbica de I; N é o fotoperíodo diário (horas); e ND o número de dias do período (dias).

b) Thornthwaite modificado por Camargo (EToTmC) (CAMARGO, 1999), obtido pela Eq. (3):

EToTmC $=16 .(10 . T e f / I)^{a} .(N / 12 . N D / 30)$

em que: Tef é a temperatura efetiva diária $\left({ }^{\circ} \mathrm{C}\right)$, conforme

Tabela 1 - Número de dias úteis empregados na análise mensal de ETo, para Palmeira das Missões (PM) e Frederico Westphalen (FW), no estado do Rio Grande do Sul Santa Maria (RS), 2011.

\begin{tabular}{lccll}
\hline & \multicolumn{2}{c}{ - } & & \\
Mês & PM & FW & PM & FW \\
\hline Jan & 18 & 23 & 18 & 18 \\
Fev & 18 & 21 & 18 & 13 \\
Mar & 21 & 20 & 21 & 25 \\
Abr & 16 & 15 & 23 & 25 \\
Mai & 22 & 20 & 18 & 19 \\
Jun & 16 & 14 & 13 & 17 \\
Jul & 20 & 23 & 17 & 16 \\
Ago & 19 & 16 & 18 & 16 \\
Set & 20 & 19 & 13 & 14 \\
Out & 14 & 12 & 19 & 19 \\
Nov & 21 & 21 & 9 & 12 \\
Dez & 21 & 23 & 14 & 19 \\
Ano & 226 & 227 & 201 & 213 \\
\hline
\end{tabular}

Eq. (4): Tef=0,36.(3.Tmax-Tmin)

em que: Tmax é a temperatura máxima do ar $\left({ }^{\circ} \mathrm{C}\right)$; e Tmin a temperatura mínima do $\operatorname{ar}\left({ }^{\circ} \mathrm{C}\right)$.

c) Camargo (EToC) (CAMARGO, 1971), obtido pela Eq. (5): $\mathrm{EToC}=$ F.Qo.T.ND

em que: Qo é a radiação solar extraterrestre $\left(\mathrm{mm} \mathrm{d}^{-1}\right)$; e $\mathrm{F}$ o fator de ajuste em função da temperatura média anual do local, igual a 0,01 .

d) Hargreaves \& Samani (EToH) (HARGREAVES \& SAMANI, 1985), calculado pela Eq. (6):

$\mathrm{EToH}=0,0023$. Qo. $(\text { Tmax-Tmin })^{0,5} .(\mathrm{T}+17,8)$

em que: Tmax é a temperatura máxima do $\operatorname{ar}\left({ }^{\circ} \mathrm{C}\right)$; e Tmin a temperatura mínima do $\operatorname{ar}\left({ }^{\circ} \mathrm{C}\right)$.

e) Makking (EToMk) (MAKKING, 1957), calculado pela Eq. (7):

$\mathrm{EToMK}=0,61$. W.Rs $-0,12$

$\mathrm{W}=0,407+0,0145 . \mathrm{T}$ para $0^{\circ} \mathrm{C}<\mathrm{T}<16^{\circ} \mathrm{C}$

$\mathrm{W}=0,483+0,01 . \mathrm{T}$ para $16,1^{\circ} \mathrm{C}<\mathrm{T}<32^{\circ} \mathrm{C}$

em que: $\mathrm{W}$ é o fator de ponderação, função de $\mathrm{T}$, calculado pelas Eq. 8 ou 9; Rs a radiação solar global incidente $\left(\mathrm{mm} \mathrm{d}^{-1}\right)$.

f) Radiação FAO-24 (EToRs), conforme descrito por PEREIRA et al. (1997), obtido pela Eq. (10):

EToRs $=-0,3+$ cl.W.Rs

$\mathrm{cl}: \mathrm{a}_{1}+\mathrm{a}_{2}$

$\mathrm{cl}=\mathrm{ao}+\mathrm{alUR}+\mathrm{a} 2 \mathrm{Ud}+\mathrm{a} 3 \mathrm{UR} \mathrm{Ud}+\mathrm{a} 4 \mathrm{UR}^{2}+\mathrm{a} 5 \mathrm{Ud}^{2}$

$\mathrm{co}=-0,3 \mathrm{~mm} \mathrm{~d}^{-1}$ ao $=1,0656$ al $=-0,0012795$ a2 $=$ 0,044953 a3 $=-0,00020033$ a4 $=-0,000031508$ a5 $=$ 0,0011026 , em que: UR é a umidade relativa média do ar (\%); e Ud a velocidade média do vento a $2 \mathrm{~m}\left(\mathrm{~m} \mathrm{~s}^{-1}\right)$

A avaliação do desempenho foi conduzida através do ajuste de modelos de regressão entre os diferentes métodos de estimação da evapotranspiração, em relação ao método de Penman-Monteith (FAO), tomado como referência, e do valor do coeficiente de correlação, do coeficiente de exatidão - índice de Willmott "d" e do coeficiente de confiança ou desempenho "c" descrito por CAMARGO \& SENTELHAS (1997). Os valores de "c" foram classificados em ótimo (OT.) para valores maiores que 0,86 ; muito bom (MB.) entre 0,76 a 0,85 ; bom (B.) entre 0,66 a 0,75 ; regular (RG.) para valores entre 0,51 a 0,65 ; ruim (RM.) entre 0,41 a 0,50 ; e péssimo (PE.) para valores menores que 0,4 .

\section{RESULTADOS E DISCUSSÃO}

Na tabela 2, são apresentados os valores dos coeficientes a e b dos modelos de regressão e os respectivos coeficientes de determinação $\left(\mathrm{R}^{2}\right)$ para os diferentes métodos de estimativa de ETo, tomando-se como referência o método de Penman-Monteith (FAO) (EToPM). Observa-se que, para Frederico Westphalen, em todas as escalas temporais testadas, o método da 
Tabela 2 - Resultados da análise de regressão da ETo nas escalas diária e de 5, 10, 15 e 30 dias, calculada pelos métodos descritos abaixo, em relação a ETo calculada por Penman-Monteith (FAO), para Frederico Westphalen e Palmeira das Missões, no estado do Rio Grande do Sul. Santa Maria (RS), 2011.

\begin{tabular}{|c|c|c|c|c|c|c|c|}
\hline Escala & Coef. & EToHS & EToC & EToTh & EToTmC & EToMk & EToRs \\
\hline & & 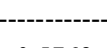 & ederico W & len-RS -- & - & 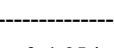 & - \\
\hline \multirow{3}{*}{ Diária } & $\mathrm{a}$ & 0,5769 & 0,1928 & $-1,2730$ & $-0,1758$ & $-0,1654$ & $-0,9361$ \\
\hline & $\mathrm{b}$ & 0,7375 & 0,7028 & 1,2518 & 0,9016 & 0,8433 & 1,4088 \\
\hline & $\mathrm{R}^{2}$ & 0,5848 & 0,6964 & 0,4885 & 0,6216 & 0,9344 & 0,9823 \\
\hline \multirow{3}{*}{5 Dias } & $\mathrm{a}$ & $-0,6180$ & $-0,3021$ & $-0,8077$ & $-0,6067$ & $-0,1778$ & $-0,7424$ \\
\hline & $\mathrm{b}$ & 0,9780 & 0,7151 & 0,7533 & 0,9772 & 0,8418 & 1,2990 \\
\hline & $\mathrm{R}^{2}$ & 0,8467 & 0,8145 & 0,6225 & 0,7078 & 0,9431 & 0,9476 \\
\hline \multirow{3}{*}{10 Dias } & $\mathrm{a}$ & $-0,2072$ & $-0,5690$ & $-1,0513$ & $-0,6909$ & $-0,2627$ & $-0,8661$ \\
\hline & $\mathrm{b}$ & 1,0115 & 0,7754 & 0,8022 & 0,9837 & 0,8600 & 1,3282 \\
\hline & $\mathrm{R}^{2}$ & 0,8818 & 0,8767 & 0,6684 & 0,6926 & 0,9489 & 0,9351 \\
\hline \multirow{3}{*}{15 Dias } & $\mathrm{a}$ & $-0,3378$ & $-0,6351$ & $-1,2072$ & $-0,8571$ & $-0,2651$ & $-0,8295$ \\
\hline & $\mathrm{b}$ & 1,0437 & 0,7904 & 0,8416 & 1,0287 & 0,8610 & 1,3156 \\
\hline & $\mathrm{R}^{2}$ & 0,8975 & 0,8992 & 0,7113 & 0,7198 & 0,9541 & 0,9468 \\
\hline \multirow{3}{*}{30 Dias } & a & $-0,4844$ & $-0,8851$ & $-1,5067$ & $-0,9569$ & $-0,3802$ & $-0,9212$ \\
\hline & $\mathrm{b}$ & 1,0738 & 0,8479 & 0,9065 & 1,0381 & 0,8968 & 1,3474 \\
\hline & $\mathrm{R}^{2}$ & 0,9061 & 0,9163 & 0,7028 & 0,6852 & 0,9571 & 0,9362 \\
\hline \multirow{3}{*}{ Diária } & $\mathrm{a}$ & 0,9154 & $\begin{array}{c}\text { Imeira da } \\
0,0143\end{array}$ & $\begin{array}{c}\text { ões-RS -- } \\
-0,1142\end{array}$ & 0,4409 & $-0,0420$ & $-0,6881$ \\
\hline & $\mathrm{b}$ & 0,6760 & 0,6289 & 0,6251 & 0,6973 & 0,8356 & 1,3465 \\
\hline & $\mathrm{R}^{2}$ & 0,6434 & 0,6990 & 0,6567 & 0,7091 & 0,8877 & 0,9451 \\
\hline \multirow{3}{*}{5 Dias } & $\mathrm{a}$ & 0,1824 & $-0,2456$ & $-0,4939$ & 0,1083 & $-0,3355$ & $-0,9240$ \\
\hline & $\mathrm{b}$ & 0,8733 & 0,6666 & 0,7047 & 0,7645 & 0,9030 & 1,3954 \\
\hline & $\mathrm{R}^{2}$ & 0,8270 & 0,8010 & 0,7588 & 0,8076 & 0,9398 & 0,9643 \\
\hline \multirow{3}{*}{10 Dias } & a & $-0,0821$ & $-0,4583$ & $-0,6514$ & $-0,0653$ & $-0,4236$ & $-0,9352$ \\
\hline & $\mathrm{b}$ & 0,9350 & 0,7176 & 0,7402 & 0,8022 & 0,9225 & 1,3953 \\
\hline & $\mathrm{R}^{2}$ & 0,8752 & 0,8553 & 0,8135 & 0,8500 & 0,9563 & 0,9706 \\
\hline \multirow{3}{*}{15 Dias } & $\mathrm{a}$ & $-0,3009$ & $-0,5824$ & $-0,7001$ & $-0,1394$ & $-0,4900$ & $-0,9541$ \\
\hline & $\mathrm{b}$ & 0,9846 & 0,7454 & 0,7495 & 0,8178 & 0,9361 & 1,3954 \\
\hline & $\mathrm{R}^{2}$ & 0,8981 & 0,8515 & 0,7737 & 0,8452 & 0,9625 & 0,9735 \\
\hline \multirow{3}{*}{30 Dias } & $\mathrm{a}$ & $-0,5267$ & $-0,7575$ & $-0,8538$ & $-0,2643$ & $-0,5440$ & $-0,9736$ \\
\hline & $\mathrm{b}$ & 1,0319 & 0,7792 & 0,7761 & 0,8412 & 0,9465 & 1,4019 \\
\hline & $\mathrm{R}^{2}$ & 0,9260 & 0,9078 & 0,8280 & 0,8567 & 0,9713 & 0,9736 \\
\hline
\end{tabular}

Método de Hargreaves \& Samani (EToHS), método de Camargo (EToC), método de Thornthwaite (EToTh), método de Thornthwaite modificado por Camargo (EToTmC), método de Makking (EToMk) e método da Radiação-FAO (EToRs).

a é o coeficiente linear, b o coeficiente angular e $\mathrm{R}^{2}$ o coeficiente de determinação gerados na análise de regressão.

Radiação-FAO 24 superestimou os valores de ETo (EToPM). A mesma resposta foi constatada na escala diária com o método de Thornthwaite (EToTh) e nas escalas de 10, 15 e 30 dias com o método de Hargreaves \& Samani (EToHS). Em Palmeira das Missões, para todas as escalas temporais avaliadas, apenas o método da Radiação FAO-24 (EToRs) apresentou superestimativa dos valores de ETo (EToPM).

Na escala diária (Tabela 3), para Frederico Westphalen, os métodos de Makking (EToMk) e da Radiação FAO24 (EToRS) apresentaram desempenho ótimo, com valores de "c" iguais a 0,88 e 0,91 
Tabela 3 - Coeficientes de correlação (r), exatidão (d) e de desempenho (c) para a ETo calculada nas escalas diária e de 5, 10, 15 e 30 dias, pelos métodos abaixo descritos, em comparação a ETo calculada por Penman-Monteith (FAO), para Frederico Westphalen e Palmeira das Missões, no estado do Rio Grande do Sul. Santa Maria (RS), 2011.

\begin{tabular}{|c|c|c|c|c|c|c|c|}
\hline & Coef. & EToHS & EToC & EToTh & EToTmC & EToMk & EToRs \\
\hline \multirow{3}{*}{ Diária } & $\mathrm{r}$ & 0,75 & 0,83 & 0,68 & 0,79 & 0,97 & 0,98 \\
\hline & $\mathrm{d}$ & 0,85 & 0,8 & 0,75 & 0,85 & 0,91 & 0,92 \\
\hline & $\mathrm{c}$ & 0,63/RG. & $0,66 / \mathrm{B}$ & 0,51/RM. & 0,67/B. & 0,88/OT. & $0,91 / \mathrm{OT}$ \\
\hline \multirow{3}{*}{5 Dias } & $\mathrm{r}$ & 0,92 & 0,9 & 0,77 & 0,83 & 0,97 & 0,97 \\
\hline & $\mathrm{d}$ & 0,95 & 0,71 & 0,65 & 0,86 & 0,89 & 0,94 \\
\hline & $\mathrm{c}$ & $0,88 / \mathrm{OT}$ & 0,64/RG. & 0,50/RM. & 0,72/B. & $0,86 / \mathrm{OT}$ & $0,91 / \mathrm{OT}$ \\
\hline \multirow{3}{*}{10 Dias } & $\mathrm{r}$ & 0,94 & 0,92 & 0,78 & 0,82 & 0,97 & 0,96 \\
\hline & d & 0,96 & 0,71 & 0,64 & 0,84 & 0,88 & 0,93 \\
\hline & $\mathrm{c}$ & 0,90/OT. & $0,66 / \mathrm{B}$ & 0,50/RM. & 0,69/B. & $0,85 / \mathrm{OT}$ & 0,89/OT. \\
\hline \multirow{3}{*}{15 Dias } & $\mathrm{r}$ & 0,95 & 0,93 & 0,8 & 0,85 & 0,97 & 0,96 \\
\hline & d & 0,97 & 0,72 & 0,65 & 0,86 & 0,88 & 0,93 \\
\hline & c & 0,92/OT. & 0,67/B. & 0,52/RG. & 0,73/MB. & 0,86/OT. & 0,90/OT. \\
\hline \multirow{3}{*}{30 Dias } & $\mathrm{r}$ & 0,95 & 0,93 & 0,78 & 0,81 & 0,97 & 0,96 \\
\hline & $\mathrm{d}$ & 0,97 & 0,7 & 0,61 & 0,82 & 0,88 & 0,91 \\
\hline & $\mathrm{c}$ & 0,92/OT. & 0,65/RG. & 0,48/RM. & $0,67 / \mathrm{B}$ & 0,85/OT. & $0,88 / \mathrm{OT}$ \\
\hline \multirow{3}{*}{ Diária } & $\mathrm{r}$ & 0,77 & $\begin{array}{l}0,84 \\
0,-P a l m e i r a \\
\end{array}$ & $\begin{array}{c}\text { Missões-RS } \\
0,81\end{array}$ & 0,83 & 0,94 & 0,97 \\
\hline & $\mathrm{d}$ & 0,87 & 0,71 & 0,69 & 0,84 & 0,91 & 0,92 \\
\hline & $\mathrm{c}$ & 0,66/B & 0,59/RG. & 0,56/RG. & 0,70/B. & 0,86/OT. & 0,89/OT. \\
\hline \multirow{3}{*}{5 Dias } & $\mathrm{r}$ & 0,91 & 0,89 & 0,86 & 0,9 & 0,97 & 0,97 \\
\hline & $\mathrm{d}$ & 0,94 & 0,67 & 0,66 & 0,84 & 0,91 & 0,91 \\
\hline & $\mathrm{c}$ & 0,85/OT. & 0,60/RG. & 0,57/RG. & 0,75/B. & 0,88/OT. & $0,89 / \mathrm{OT}$ \\
\hline \multirow{3}{*}{10 Dias } & $\mathrm{r}$ & 0,94 & 0,92 & 0,88 & 0,92 & 0,97 & 0,97 \\
\hline & $\mathrm{d}$ & 0,95 & 0,68 & 0,66 & 0,84 & 0,91 & 0,91 \\
\hline & c & 0,89/OT. & $0,62 / \mathrm{RG}$ & 0,59/RG. & 0,78/MB. & 0,89/OT. & 0,89/OT. \\
\hline \multirow{3}{*}{15 Dias } & $\mathrm{r}$ & 0,95 & 0,91 & 0,86 & 0,92 & 0,97 & 0,98 \\
\hline & $\mathrm{d}$ & 0,95 & 0,67 & 0,65 & 0,83 & 0,91 & 0,91 \\
\hline & c & 0,90/OT. & $0,61 / \mathrm{RG}$ & 0,56/RG. & 0,76/MB. & 0,88/OT. & 0,89/OT. \\
\hline \multirow{3}{*}{30 Dias } & $\mathrm{r}$ & 0,96 & 0,93 & 0,88 & 0,92 & 0,98 & 0,97 \\
\hline & $\mathrm{d}$ & 0,96 & 0,66 & 0,64 & 0,83 & 0,9 & 0,9 \\
\hline & $\mathrm{c}$ & 0,92/OT. & 0,61/RG. & 0,56/RG. & 0,76/MB. & 0,88/OT. & $0,88 / \mathrm{OT}$ \\
\hline
\end{tabular}

Método de Hargreaves \& Samani (EToHS), método de Camargo (EToC), método de Thornthwaite (EToTh), método de Thornthwaite modificado por Camargo (EToTmC), método de Makking (EToMk) e método da Radiação-FAO (EToRs).

respectivamente, superando os métodos de Camargo (EToC), $(\mathrm{c}=0,66)$, e Thornthwaite modificado por Camargo (EToTmC), $(\mathrm{c}=0,66)$, classificados como bom. $\mathrm{Na}$ mesma escala temporal (Tabela 3), para Palmeira das Missões, os métodos de Makking (EToMk) e Radiação FAO24 (EToRS) obtiveram classe ótimo, com valores de "c" iguais a 0,86 e 0,89 , respectivamente.
Os desempenhos de Camargo (EToC) e Thornthwaite (EToTh) para Palmeira das Missões, e de Thornthwaite (EToTh) para Frederico Westphalen, demonstram a limitação desses métodos para uso em escala diária nessas localidades (Tabela 3). No entanto, resultados melhores desses dois métodos foram obtidos para a região de Palotina, PR (SYPERRECK et 
al., 2008), e no estado de São Paulo (CAMARGO \& SENTELHAS, 1997).

Considerando ainda a escala diária, resultados para os métodos de Makking (EToMk) e Radiação FAO24 (EToRS), que incorporam a radiação solar incidente (Rs) como variável de entrada, são mais precisos e exatos, como também observado por CONCEIÇÃO \& MANDELLI (2005) no estado do Rio Grande do Sul. Já para alguns locais do Estado de São Paulo, CAMARGO \& SENTELHAS (1997) observaram resultados diferenciados, em que métodos que utilizam apenas dados de temperatura do ar se destacaram em relação a esses dois.

Hargreaves \& Samani (EToHS) e Thornthwaite modificado por Camargo (EToTmC) foram os métodos que mais se aproximaram dos métodos que utilizam a radiação solar (Rs) como variável de entrada, com valor de "c" superior 0,63 para ambas as localidades, na escala diária (Tabela 3). O desempenho verificado para EToHS concorda com os resultados observados para localidades como Bento Gonçalves, RS (CONCEIÇÃO \& MANDELLI, 2005), Santa Maria, RS (MEDEIROS, 1998), Baixo Rio Grande, SP (CONCEIÇÃO, 2003), Dourados, MS (FIETZ, 2005) e Palotina, PR (SYPERRECK et al., 2008).

Em intervalos de 5 dias (Tabela 3), em Frederico Westphalen, os métodos avaliados apresentaram desempenho igual ou superior aos obtidos na escala diária, mantendo a classe mínima "ruim" para EToTh. Em análises decendiais ou quinzenais, os métodos EToHS, EtoMk e EToRs equipararam-se com ótimo desempenho. Nessas escalas temporais, os melhores desempenhos foram obtidos com os métodos EToTh e EToTmC. Na transição entre as escalas diária e de cinco dias, para Palmeira das Missões, também observou-se manutenção ou melhora de desempenho de todos os métodos, com classificação mínima de regular. Para as escalas decendial e quinzenal, os resultados foram semelhantes, novamente com destaque para EToHS, EToMk e EToRs, pelo ótimo desempenho.

$\mathrm{Na}$ escala de 30 dias, tanto para Frederico Westphalen quanto para Palmeira das Missões, os métodos de estimativa da ETo apresentaram praticamente o mesmo desempenho demonstrado para as análises decendiais e quinzenais. As exceções foram os métodos de Thornthwaite (EToTh) e Camargo (EToC), que obtiveram classificação inferior, entre ruim e regular (Tabela 3), para Frederico Westphalen. Em Urussanga, SC, BACK (2008), em escala mensal, obteve desempenho muito bom para EToC e EToTh, bom para EToTmC e péssimo para EToHS, contrário aos resultado encontrados. Apesar do desenvolvimento do método de Thornthwaite basear-se em dados de lisímetros, na escala de 30 dias (PEREIRA et al., 1997), seu desempenho inferior pode ser atribuído pelo ajuste do brilho solar para 12 horas, resultando em perda de desempenho em escalas menores de tempo ou de brilho solar.

Avaliando o desempenho dos métodos propostos para estimativa da evapotranspiração de referência frente ao método de Penman-Monteith (FAO) (EToPM), para cada mês do ano (Tabela 1), verifica-se que o valor mínimo obtido a partir de EToPM ficou muito próximo aos $2,00 \mathrm{~mm} \mathrm{~d}^{-1}$, em junho, enquanto, no mês de dezembro, a EToPM ultrapassou os 6,00 mm.d $\mathrm{d}^{-1}$, em ambas as localidades (Figura 1A, 1C).

Tomando os valores de ETo calculados pelo método de Penman-Monteith (FAO) (EToPM) como padrão, igual a 1 ou $100 \%$, e comparando aos resultados dos demais modelos testados, observa-se que o método EToRs tendeu a superestimar a EToPM na maior parte do ano. Exceção para os meses de maio, junho e julho, incluindo subestimação de ETo tanto para Frederico Westphalen quanto para Palmeira das Missões (Figura 1B, 1D). Os demais métodos apresentaram tendência de subestimar EToPM na maioria dos meses. Ressalvas para os resultados do método de EToTh, que, entre os meses de novembro e abril, superestimou a EToPM em Frederico Westphalen.

Resultados similares foram obtidos por MENDONÇA et al. (2003), que destacam valores subestimados pelo método de Makking e superestimados pelo método da Radiação FAO-24, em comparação a ETo calculada a partir de PenmanMonteith para Campo dos Goytacazes, RJ. FIETZ (2005), em estudo realizado para Dourados, MS, observou uma superestimativa do método de Makking, resposta que pode estar associada aos coeficientes originais da equação, por serem desenvolvidos para a Holanda (CUNHA \& BERGAMASCHI, 1994).

\section{CONCLUSÃO}

Para qualquer uma das escalas temporais avaliadas, os métodos de Makking e da Radiação FAO24, que incluem dados de radiação solar incidente como variável de entrada, podem substituir o método de Penman-Monteith (FAO). Dispondo-se apenas de dados de temperatura do ar para o cálculo da ETo, na escala diária, os métodos de Camargo (EToC) e Thornthwaite modificado por Camargo (EToTmC) para Frederico Westphalen, e de Hargreaves \& Samani (EToHS) e Thornthwaite modificado por Camargo (EToTmC) para Palmeira das Missões, devem preferencialmente serem adotados. 


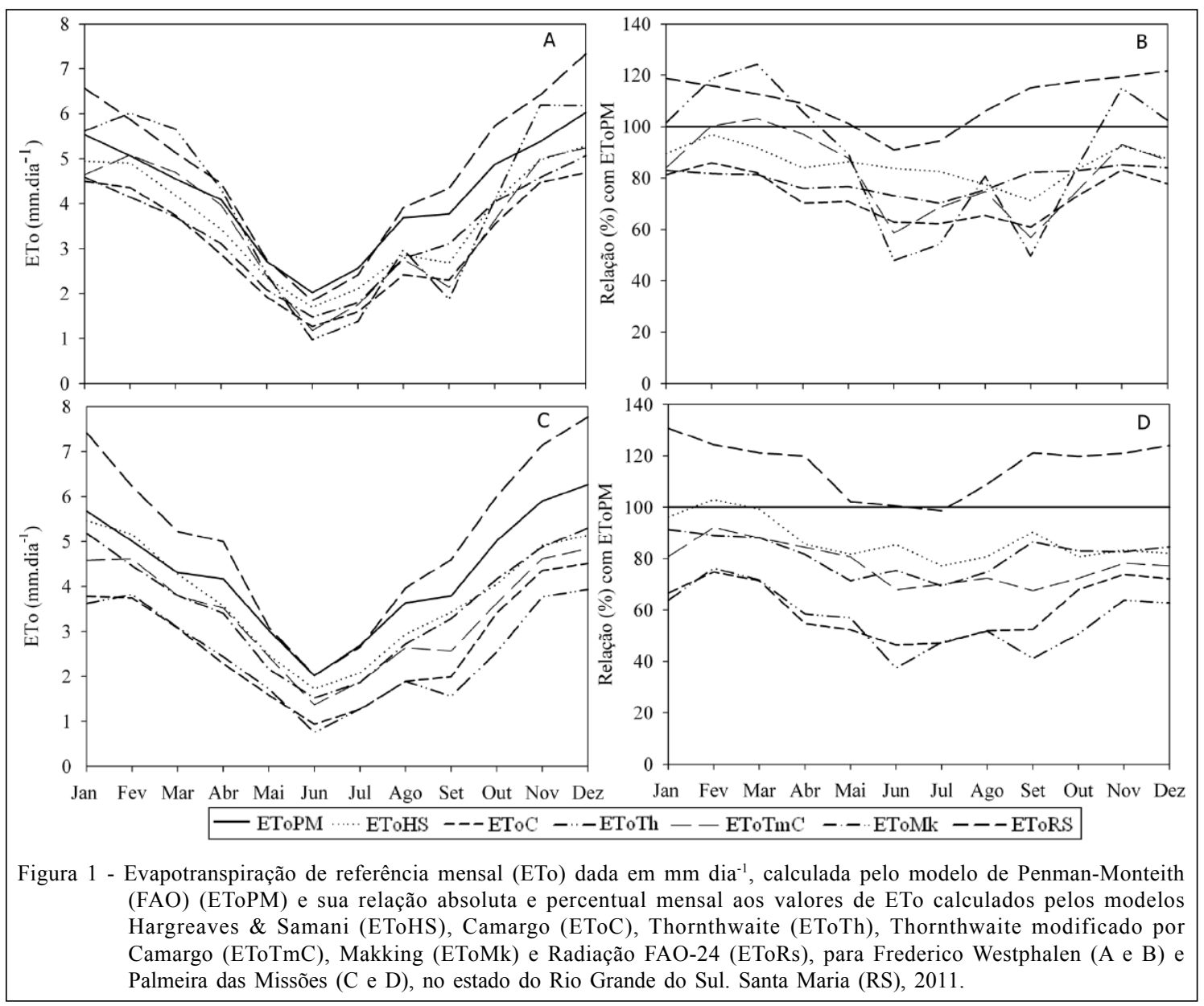

\section{REFERÊNCIAS}

ALLEN, R.G. et al. Crop evapotranspiration - Guidelines for computing crop water requirements. Roma: FAO Irrigation and drainage, 1998. 297p. (Paper 56). Disponível em: <http://www.fao.org/docrep/X0490E/X0490E00.htm>. Acesso em: 15 out. 2009.

BACK, A.J. Desempenho de métodos empíricos baseados na temperatura do ar para a estimativa da evapotranspiração de referencia em Urussanga, SC. Irriga, v.13, n.4, p.449466, 2008. Disponível em: <http://200.145.140.50/ojs1/ viewarticle.php?id=367\&layout $=$ abstract $>$. Acesso em: 15 out. 2009 .

BERLATO, M.A. et al. Associação entre El Niño Oscilação Sul e a produtividade do milho no Estado do Rio Grande do Sul. Pesquisa Agropecuária Brasileira, v.40, n.5, p.423-432, 2005. Disponível em: <http://www.scielo.br/pdf/pab/v40n5/ 24422.pdf $>$. Acesso em: 15 out. 2009. doi: 10.1590/S0100$204 X 2005000500001$.

CAMARGO, A.P.; SENTELHAS, P.C. Avaliação do desempenho de diferentes métodos de estimativa da evapotranspiração potencial no estado de São Paulo, Brasil. Revista Brasileira de Agrometeorologia, v.5, n.1, p.89-97, 1997.
CAMARGO, A.P. Balanço hídrico no Estado de São Paulo. 3.ed. Campinas: Instituto Agronômico, 1971. 24p. (Boletim, 116).

CAMARGO, A.P. et al. Ajuste da equação de Thornthwaite para estimar a evapotranspiração potencial em climas áridos e superúmidos, com base na amplitude térmica diária. Revista Brasileira de Agrometeorologia, v.7, n.2, p.252-257, 1999.

COMPANHIA NACIONAL DE ABASTECIMENTO (CONAB). Disponível em: <http://www.conab.gov.br/conabweb/ index.php?PAG=131>. Acesso em: 01 out. 2009.

CONCEIÇÃO, M.A.F. Estimativa da evapotranspiração de referência com base na temperatura do ar para as condições do Baixo Rio Grande, SP. Revista Brasileira de Agrometeorologia, v.11, n.2, p.229-236, 2003.

CONCEIÇÃO, M.A.F.; MANDELLI, F. Comparação entre métodos de estimativa da evapotranspiração de referência em Bento Gonçalves, RS. Revista Brasileira de Agrometeorologia, v.13, n.2, p.303-307, 2005.

CUNHA, G.R.; BERGAMASCHI, H. Coeficientes das equações de Making e Priestley para estimativa da evapotranspiração máxima de alfafa. Revista Brasileira de Agrometeorologia, v.2, p.33-36, 1994.

Ciência Rural, v.42, n.2, fev, 2012. 
FIETZ, C. R. et al. Estimativa da evapotranspiração de referência diária para a região de Dourados, MS. Revista Brasileira de Agrometeorologia, v.13, n.2, p.225-250, 2005.

HARGREAVES, G.H.; SAMANI, Z.A. Reference crop evapotranspiration from temperature. Applied Engineering Agriculture, v.1, n.2, p.96-99, 1985.

MAKKING, G.F. Ekzameno de la formulo de Penman. Netherlands Journal of Agricultural Science, n.5, p.290$305,1957$.

MEDEIROS, S.L.P. Avaliação de métodos de estimativa da evapotranspiração de referência para a região mesoclimática de Santa Maria-RS. Revista Brasileira de Agrometeorologia, v.6, n.1, p.105-109, 1998.

MENDONÇA, J.C. et al. Comparação entre métodos de estimativa da evapotranspiração de referência (ETo) na região Norte Fluminense, RJ. Revista Brasileira de Engenharia Agrícola e Ambiental, v.7, n.2, p.275-279, 2003. Disponível em: <http://www.scielo.br/pdf/rbeaa/v7n2/v7n2a15.pdf $>$. Acesso em: 15 out. 2010 . doi: 10.1590/S1415-43662003000200015.

MORENO, J.A. Clima do Rio Grande do Sul. Porto Alegre: Secretaria da Agricultura, 1961. 41p.
PEREIRA, A.R. et al. Evapo(transpi)ração. Piracicaba: FEALQ, 1997. 183p.

REIS, E.F. dos et al. Estudo comparativo da estimativa da evapotranspiração de referência para três localidades do Estado do Espírito Santo no período seco. IDESIA, v.25, n.3, p.7584, 2007. Disponível em: <http://www.scielo.cl/pdf/idesia/ v25n3/art08.pdf>. Acesso em: 15 out. 2009. doi: 10.4067/ S0718-34292010000200003.

SILVA, K.O. da et al. Análise de métodos de estimativa de evapotranspiração na otimização de sistemas de drenagem. Revista Brasileira de Engenharia Agrícola e Ambiental, v.9, n.2, p.161-165, 2005. Disponível em: <http://www.scielo.br/ pdf/rbeaa/v9n2/v9n2a02.pdf>. Acesso em: 15 out. 2009. doi: $10.1590 / \mathrm{S} 1415-43662005000200002$.

SYPERRECK, V.L.G. et al. Avaliação de desempenho de métodos para estimativas de evapotranspiração de referência para a região de Palotina, Estado do Paraná. Acta Scientiarum Agronomy, v.30, p.603-609, 2008. Disponível em: <http://periodicos.uem.br/ ojs/index.php/ActaSciAgron/article/view/5959/5959>. Acesso em: 15 out. 2009 . doi: $10.4025 /$ ct sci gron.v30i5.5959.

THORNTHWAITE, C.W. An approach toward a rational classification of climate. Geographical Review, v.38, p.5594, 1948 . 